\title{
Recovery of Renal Function in a Dialysis-dependent Patient with Microscopic Polyangiitis and both Myeloperoxidase Anti-neutrophil Cytoplasmic Antibodies and Anti-glomerular Basement Membrane Antibodies
}

\author{
Shintaro Mandai ${ }^{1}$, Kiyotaka Nagahama ${ }^{2}$, Yukio Tsuura ${ }^{3}$, Toshiyuki Hirai ${ }^{1}$, \\ Wakako Yoshioka ${ }^{1}$, Daiei Takahashi ${ }^{1}$, Shota $\mathrm{Aki}^{1}{ }^{1}$, Makoto Aoyagi ${ }^{1}$, \\ Hiroyuki Tanaka $^{1}$ and Teiichi Tamura ${ }^{1}$
}

\begin{abstract}
A 40-year-old man with microscopic polyangiitis developed both myeloperoxidase anti-neutrophil cytoplasmic antibodies (90 EU) and anti-glomerular basement membrane antibodies (134 EU)-positive rapidly progressive glomerulonephritis and heparin-induced thrombocytopenia. Although the patient initially showed no signs of improvement, persistent therapy including $1 \mathrm{~g} /$ day intravenous methylprednisolone, $50 \mathrm{mg} /$ day oral prednisolone, plasma exchange, and $900 \mathrm{mg} /$ day intravenous cyclophosphamide resulted in the normalization of both titers, recovery of renal function, and discontinuation of dialysis. Though previous studies showed poor outcomes in such "double-positive" patients, aggressive immunosuppression in younger patients may result in the recovery of renal function, even in those with severe renal dysfunction.
\end{abstract}

Key words: anti-GBM antibodies, HIT, microscopic polyangiitis, MPO-ANCA, RPGN

(Intern Med 50: 1599-1603, 2011)

(DOI: 10.2169/internalmedicine.50.4939)

\section{Introduction}

Myeloperoxidase anti-neutrophil cytoplasmic antibodies (MPO-ANCA) and anti-glomerular basement membrane (anti-GBM) antibodies are autoantibodies that can cause rapidly progressive glomerulonephritis (RPGN). MPO-ANCA is associated with microscopic polyangiitis (MPA), ChurgStrauss syndrome, or idiopathic necrotizing crescentic glomerulonephritis, whereas anti-GBM antibodies are associated with anti-GBM antibody disease including Goodpasture's disease. Several previous studies have reported patients with both ANCA and anti-GBM antibody-positive RPGN. The majority of these studies reported poor outcomes in such "double-positive" patients, both in terms of the recovery of renal function and mortality (1-3). The largest study showed that 1-year patient survival was $35 \%$ and renal survival was $0 \%$ in dialysis-dependent, double-positive patients (1). In this report, a patient with MPA who developed double-positive RPGN and successfully recovered renal function despite an initial need for dialysis and heparininduced thrombocytopenia (HIT) is described.

\section{Case Report}

A 40-year-old Japanese man was transferred to our hospital with acute renal dysfunction and interstitial pneumonia in April 2010. Six weeks previously, he had a moderate fever, productive cough, fatigue, and anorexia. These symptoms had worsened despite treatment with oral antibiotics including levofloxacin prescribed by his general physician. Two weeks prior to the transfer, he had visited another hospital, and the laboratory findings were as follows: hemoglobin, $11.3 \mathrm{~g} / \mathrm{dL}$; white blood cell count, $15,600 / \mu \mathrm{L}$; platelet count,

${ }^{1}$ Department of Nephrology, Yokosuka Kyosai Hospital, Japan, ${ }^{2}$ Department of Molecular Pathology, Yokohama City University, School of Medicine, Japan and ${ }^{3}$ Department of Pathology, Yokosuka Kyosai Hospital, Japan Received for publication December 3, 2010; Accepted for publication March 29, 2011 Correspondence to Dr. Shintaro Mandai, smandai@ hotmail.co.jp 


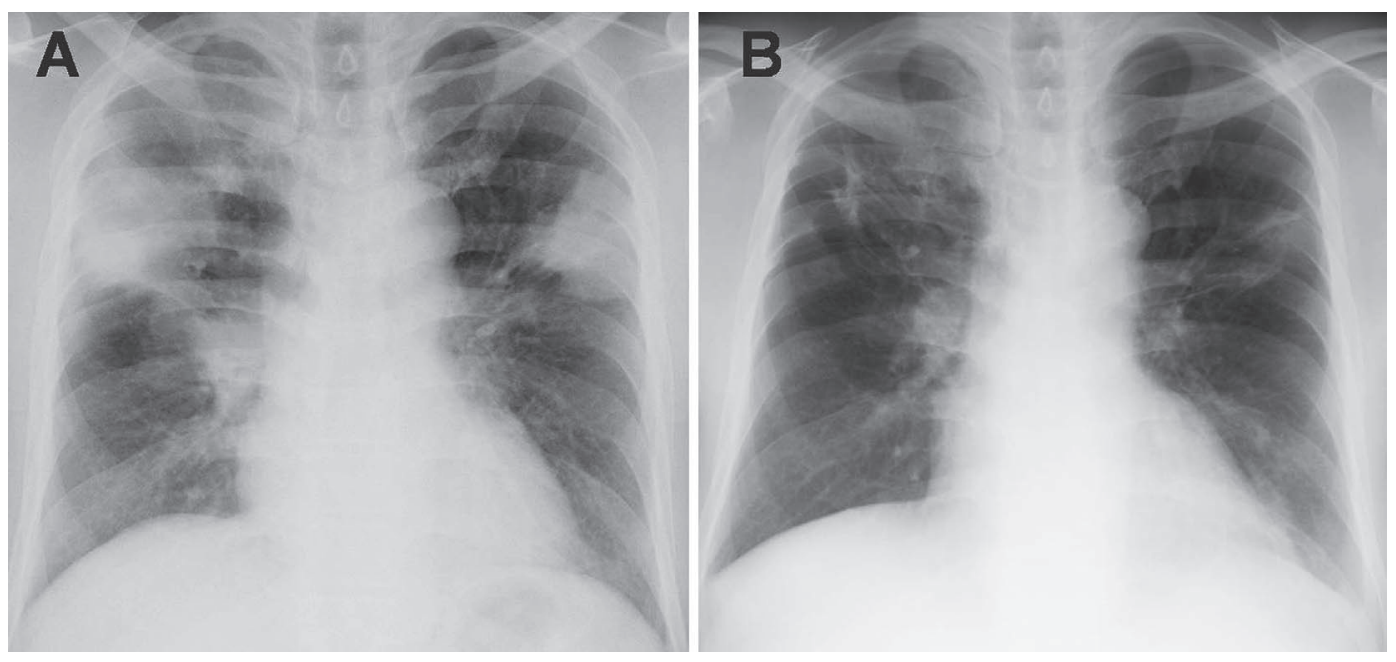

Figure 1. A chest radiograph on admission. Bilateral diffuse alveolar consolidations are seen. B Chest radiograph on discharge shows improved consolidations.

Table 1. Laboratory Data on Transfer

\begin{tabular}{|c|c|c|c|c|c|c|c|c|}
\hline Urinalysis & & & Blood chemistry & & & Serological test & & \\
\hline Protein & $2+$ & & Total protein & 7.6 & gldL & KL-6 & 575 & $\mathbf{U} / \mathbf{m L}$ \\
\hline Occult blood & $3+$ & & Albumin & 2.9 & gldL & IgG & 1615 & $\mathrm{mg} / \mathrm{dL}$ \\
\hline Red blood cells & Many & $/ \mathrm{HPF}$ & Lactate dehydrogenase & 225 & $\mathbf{U} / \mathbf{L}$ & $\mathbf{I g A}$ & 231 & $\mathrm{mg} / \mathrm{dL}$ \\
\hline White blood cells & 5-9 & /HPF & Aspartate aminotransferase & 46 & $\mathbf{U} / \mathbf{L}$ & IgM & 210 & $\mathrm{mg} / \mathrm{dL}$ \\
\hline Granular cast & Many & $/$ HPF & Alanine aminotransferase & 79 & $\mathbf{U} / \mathbf{L}$ & CH50 & $>54.0$ & $\mathbf{U} / \mathbf{m L}$ \\
\hline Complete blood count & & & Blood urea nitrogen & 68 & $\mathrm{mg} / \mathrm{dL}$ & Antinuclear antibody & $<40$ & $x$ \\
\hline White blood cells & 20400 & $/ \mu \mathrm{L}$ & Creatinine & 5.34 & $\mathrm{mg} / \mathrm{dL}$ & MPO-ANCA & 90 & $\mathbf{E U}$ \\
\hline Neutrophils & 94 & $\%$ & Sodium & 132 & $\mathrm{mEq} / \mathrm{L}$ & PR3-ANCA & $<10$ & $\mathbf{E U}$ \\
\hline Eosinophils & $\mathbf{0}$ & $\%$ & Potassium & 5.0 & $\mathrm{mEq} / \mathrm{L}$ & Anti-GBM antibody & 134 & $\mathbf{E U}$ \\
\hline Basophils & 0.5 & $\%$ & Chloride & 96 & $\mathbf{m E q} / \mathbf{L}$ & Blood gas analysis & & \\
\hline Lymphocytes & 3 & $\%$ & Calcium & 9.1 & $\mathbf{m g} / \mathbf{d L}$ & pH & 7.380 & \\
\hline Monocytes & 2.5 & $\%$ & Phosphate & 4.3 & $\mathrm{mg} / \mathrm{dL}$ & $\mathrm{PaCO}_{2}$ & 29.0 & $\mathrm{mmHg}$ \\
\hline Red blood cells & 397 & $\times 10^{4} / \mu \mathrm{L}$ & Glucose & 237 & $\mathrm{~g} / \mathrm{dL}$ & $\mathrm{PaO}_{2}$ & 106.0 & $\mathrm{mmHg}$ \\
\hline Hemoglobin & 11.5 & $\mathrm{~g} / \mathrm{dL}$ & Hemoglobin A1c & 7.0 & $\%$ & $\mathrm{HCO}_{3}^{-}$ & 16.7 & $\mathrm{mmol} / \mathrm{L}$ \\
\hline Platelets & 96.9 & $\times 10^{4} / \mu \mathrm{L}$ & C-reactive protein & 13.18 & $\mathrm{mg} / \mathrm{dL}$ & Base excess & -7.0 & $\mathrm{mmol} / \mathrm{L}$ \\
\hline
\end{tabular}

$75.9 \times 10^{4} / \mu \mathrm{L} ;$ C-reactive protein, $9.0 \mathrm{mg} / \mathrm{dL} ;$ serum creatinine, $1.02 \mathrm{mg} / \mathrm{dL}$; KL-6, $764 \mathrm{U} / \mathrm{mL}$; and slight hematuria and proteinuria. Chest radiography (Fig. 1) and computed tomography (CT) scan revealed bilateral diffuse consolidations in the upper lungs, typically seen in bronchiolitis obliterans organizing pneumonia (BOOP), a subtype of interstitial pneumonia. The patient was admitted to the hospital with a diagnosis of interstitial pneumonia and started on 40 $\mathrm{mg} /$ day oral prednisolone. After 2 weeks of hospitalization, his fever, productive cough, and consolidations on chest radiography were somewhat improved, but his serum creatinine level increased progressively to $4.28 \mathrm{mg} / \mathrm{dL}$. Therefore, he was transferred to our hospital. At the time of transfer, he noted a mild cough, fatigue, and weight loss of 14 $\mathrm{kg}$ within 3 months. His medical history included appendectomy, septoplasty, a 30-year history of asthma, a 2-year history of diabetes mellitus, hyperlipidemia, and fatty liver. On physical examination, crackles were heard bilaterally in his upper lungs. His body weight was $74 \mathrm{~kg}$, his height was $174 \mathrm{~cm}$, and his blood pressure was $138 / 87 \mathrm{mmHg}$. Laboratory findings (Table 1) were as follows: hemoglobin, $11.5 \mathrm{~g} /$
dL; white blood cell count, 20,400/ $\mu \mathrm{L}$; platelet count, $96.9 \times$ $10^{4} / \mu \mathrm{L}$; C-reactive protein, $13.18 \mathrm{mg} / \mathrm{dL}$; serum creatinine, $5.34 \mathrm{mg} / \mathrm{dL}$; MPO-ANCA, $90 \mathrm{EU}$ (normal <20 EU); antiGBM antibody, 134 EU (normal <10 EU); KL-6, 575 U/ $\mathrm{mL}$; and intense hematuria and proteinuria. Routine sputum cultures yielded no growth of microorganisms. Chest radiographs and CT scan revealed bilateral diffuse consolidations that had improved mildly compared with 2 weeks previously. A renal biopsy specimen showed 46 glomeruli, one of which was globally sclerotic. Twenty-six glomeruli showed extensive cellular crescents, and 3 glomeruli had fibrotic crescents (Fig. 2A). Severe interstitial infiltration of mononuclear leukocytes and neutrophils was noted around the crescentic glomeruli, and no thrombus was noted in the vessels. Immunofluorescence studies demonstrated no immunoglobulin or complement deposition (Fig. 2B).

A diagnosis of microscopic polyangiitis (MPA) was made on the basis of clinical symptoms, high titers of MPOANCA, and the histological findings of the renal biopsy specimen. The patient was started on $1 \mathrm{~g} /$ day intravenous methylprednisolone for 3 days, followed by $50 \mathrm{mg} /$ day oral 

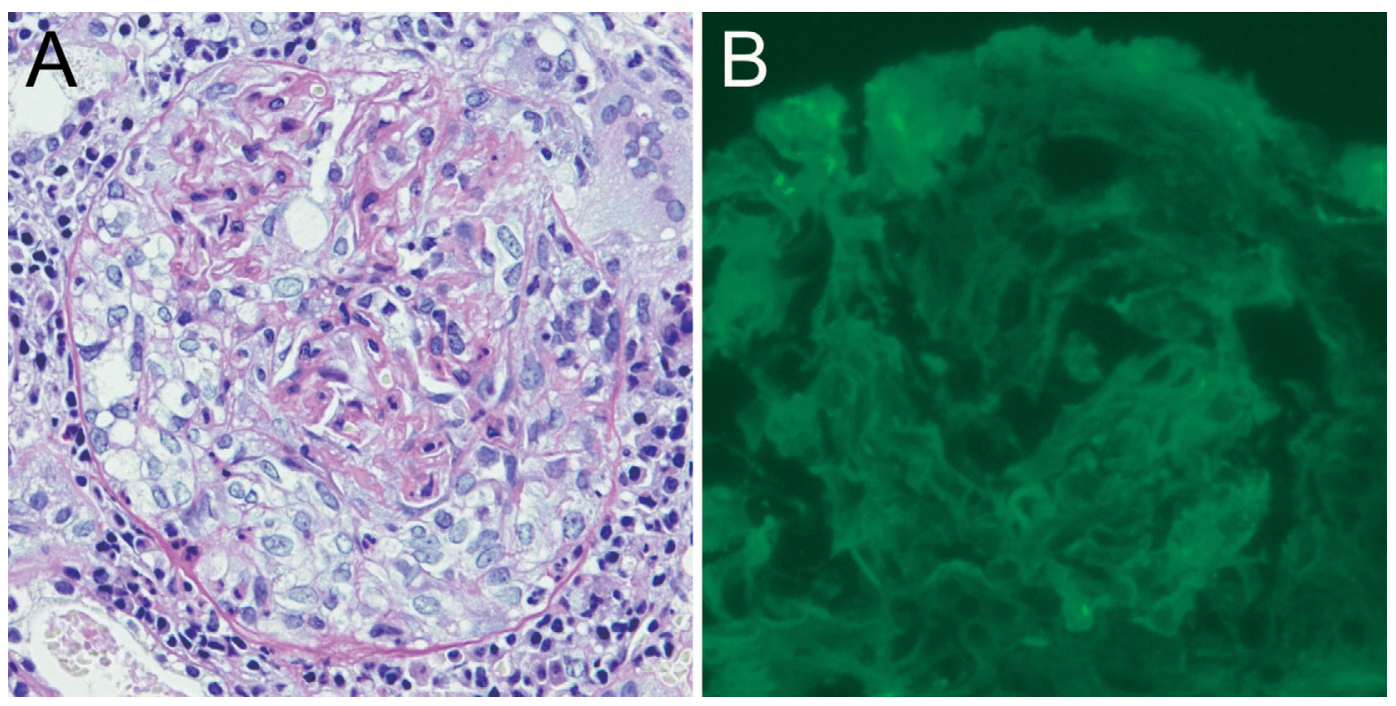

Figure 2. Light microscopy shows cellular crescentic formation (PAS stain; ×200). B Immunofluorescence microscopy shows no immunoglobulin $\mathbf{G}(\mathrm{IgG})$ deposition along the capillary walls $(\times 200)$.

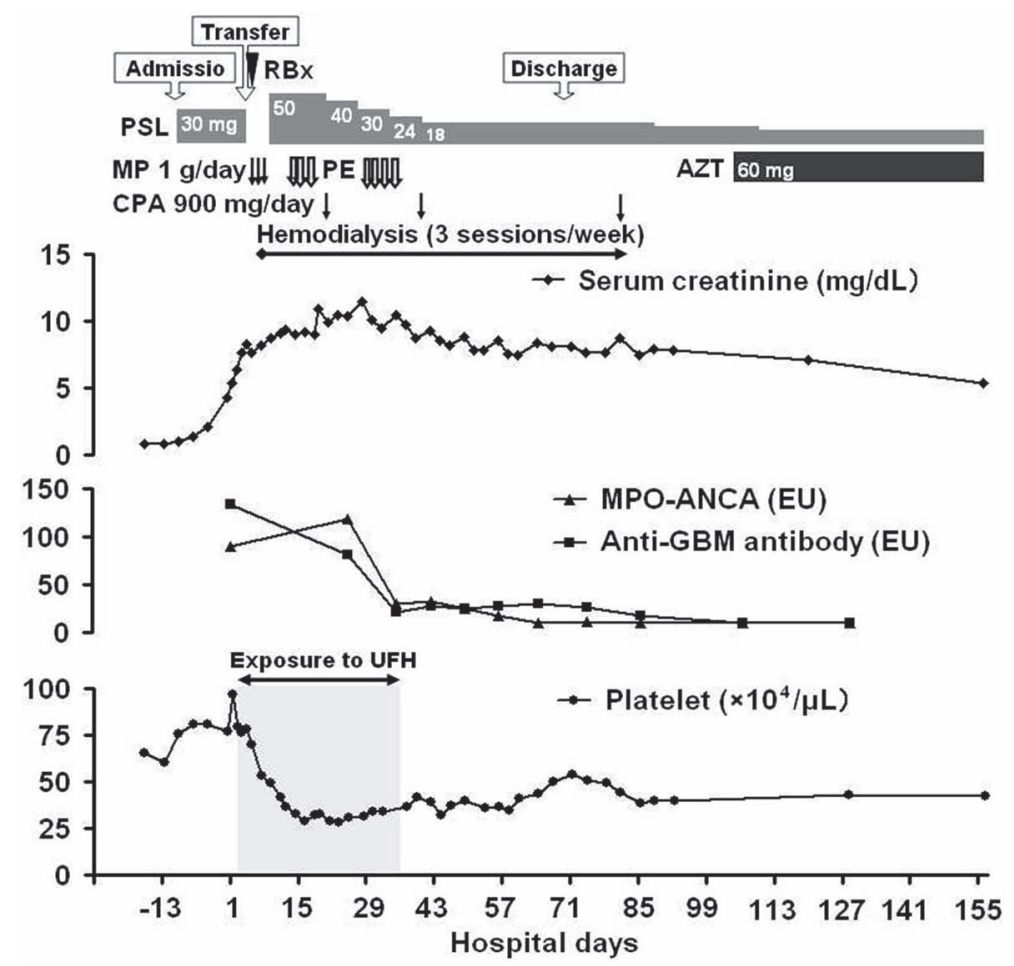

Figure 3. Clinical course. The gray area indicates the period of UFH exposure. Abbreviations: anti-GBM antibody: anti-glomerular basement membrane antibody, AZT: oral azathioprine, CPA: pulsed cyclophosphamide, MP: pulsed methylprednisolone, MPO-ANCA: myeloperoxidase antineutrophil cytoplasmic antibodies, PE: plasma exchange, PSL: oral prednisolone, RBx: renal biopsy, UFH: unfractionated heparin

prednisolone. He had oliguria, and his serum creatinine increased $1 \mathrm{mg} / \mathrm{dL}$ daily. On day 4 of hospitalization, hemodialysis had to be started, with 3 sessions weekly. Unfractionated heparin (UFH) was used as an anticoagulant during each session. Furthermore, 3-liter plasma exchange sessions with 5\%-albumin for a total of 7 sessions and $900 \mathrm{mg} /$ day intravenous cyclophosphamide for a total of 3 times were added $(4,5)$.
Five days after the first administration of UFH for line flush, on day 7 of hospitalization, his platelet count started to decrease, reaching a low of $26.9 \times 10^{4} / \mu \mathrm{L}$, a greater than $50 \%$ fall (Fig. 3). At 9 days, clotting in the dialyzer and extracorporeal circuit during dialysis and plasma exchange sessions started. Some of the sessions could not be completed, and a hemodialysis catheter needed to be changed due to clotting in the catheter. There were no other causes for 
thrombocytopenia, and HIT was suspected based on his high 4Ts clinical score (6) of 6 . Then, 26 days after the first exposure to UFH, the patient showed positive HIT antibody on enzyme-linked immunoassay (ELISA) and was diagnosed as having HIT. Heparin administration both for line flushes and hemodialysis was discontinued, and argatroban, a direct thrombin inhibitor, was used as an alternative anticoagulant during each hemodialysis session. A bolus of $5 \mathrm{mg}$ was administered at the start, followed by a continuous infusion with a dose of $15-20 \mathrm{mg} / \mathrm{hr}$, with monitoring to maintain an activated partial thromboplastin time (aPTT) value 1.5-3.0 times that at baseline. Once this treatment was started, he tolerated full hemodialysis sessions repeatedly without clotting, and his platelet count started to increase. HIT antibody became negative 3 weeks after the cessation of heparin.

His fever and productive cough disappeared several days after transfer, whereas general fatigue, anorexia, and high $\mathrm{C}$ reactive protein $(5-8 \mathrm{mg} / \mathrm{dL})$ continued. On day 18 of hospitalization, an arteriovenous fistula was created in his left arm for reliable hemodialysis access and to prevent hemodialysis catheter infection. However, in approximately 1 month, the C-reactive protein decreased to $1-2 \mathrm{mg} / \mathrm{dL}$, and his urine output started to increase, and serum creatinine and both MPO-ANCA and anti-GBM antibody titers started to decrease (Fig. 3). The maximal serum creatinine was 11.43 $\mathrm{mg} / \mathrm{dL}$. KL-6 became negative, and the consolidations on the chest radiographs improved markedly (Fig. 1B). Although improvement in fatigue and anorexia was delayed, these symptoms had almost completely disappeared at discharge on day 68 of hospitalization. At 2 weeks after discharge, his renal function became independent of dialysis and, at 4 months, his serum creatinine was less than $5.0 \mathrm{mg} /$ dL. Both MPO-ANCA and anti-GBM antibody titers turned negative at discharge and at 6 weeks after discharge, respectively. He was started on $60 \mathrm{mg} /$ day oral azathioprine to maintain remission after the third intravenous dose of cyclophosphamide.

\section{Discussion}

Several previous studies reported patients with both ANCA and anti-GBM antibody-positive RPGN. Approximately $10-20 \%$ of ANCA-positive patients also had positive anti-GBM antibodies, and 20-40\% of anti-GBM antibodypositive patients also had positive $\operatorname{ANCA}(2,7,8)$. It is unclear which antibody is the primary cause of the disease. However, Serratrice et al (9) recently suggested that glomerular damage due to ANCA-related vasculitis results in an immune reaction between anti-GBM antibodies and exposed antigen on the glomerular basement membrane. Frequently reported systemic symptoms that precede RPGN in double-positive patients, including malaise, weight loss, fever, and arthralgia, support this suggestion, since these symptoms are typical of ANCA-related vasculitis and are usually absent in anti-GBM antibody disease (2). Further, in the present case, RPGN was preceded by these complaints and interstitial pneumonia, which is typical for MPA. Although MPO-ANCA seems to be primary in the pathophysiology, the outcomes of double-positive patients are very poor both in terms of renal function recovery and mortality (1-3), presumably due to additional glomerular damage by anti-GBM antibodies.

The present patient developed MPA, with severe renal dysfunction due to MPO-ANCA and anti-GBM antibodiesassociated RPGN; the renal biopsy revealed crescentic glomerulonephritis, and more than $50 \%$ of glomeruli were affected. However, despite positive anti-GBM antibodies, immunofluorescence microscopy showed no deposition of any immunoglobulin or complement presumably due to the lower specificity of anti-GBM antibodies against the noncollagen domain 1 of the $\alpha 3$ chain of type IV collagen in double-positive patients compared with those in patients with anti-GBM antibodies alone (10). Indeed, Rutgers et al (2) revealed that $50 \%$ of double positive patients showed few immune deposits.

Optimal therapy for double-positive patients has not been identified yet. Plasma exchange and immunosuppression in addition to high-dose steroids are considered for patients with MPA even if their renal dysfunction is severe $(4,5)$, though they are not recommended, even in the Japanese guideline, for dialysis-dependent patients with anti-GBM antibody disease because of a very low likelihood of a renal response (11). On the basis of a diagnosis of MPA in the present case, aggressive therapy including plasma exchange and immunosuppression was performed while carefully monitoring for evidence of side effects. Although the patient initially exhibited no signs of improvement, with persistent therapy, renal function successfully recovered, and dialysis was discontinued. A lower titer of anti-GBM antibodies, which is possibly indicative of less glomerular damage due to anti-GBM antibodies, may be associated with a good outcome. It has been shown that a lower titer of anti-GBM antibodies was associated with increased renal survival in Goodpasture's disease (12). Furthermore, a younger age may be a predictive marker of good prognosis. In an older study that reported a better outcome in double-positive patients (13), the patients whose renal function recovered were relatively younger. In younger patients who are better able to tolerate aggressive immunosuppression, optimal therapy should include plasma exchange and immunosuppression, even if their renal dysfunction is severe. Further large studies to identify a marker of good prognosis are essential.

HIT is a syndrome typically characterized by thrombocytopenia and thrombosis, and it is mediated by anti-heparin/ platelet factor 4 antibodies in the setting of heparin administration (14). Yamamoto et al (15) reported that HIT occurred in $3.9 \%$ of patients starting hemodialysis in Japan. The prevalence of positive HIT antibody by ELISA in hemodialysis patients exposed to UFH is $8.1 \%$ worldwide (16), and positive HIT antibody is associated with increased mortality in hemodialysis patients (17). To the best of our knowledge, few cases of HIT in patients with ANCA 
vasculitis or anti-GBM antibody disease have been reported (18), and there have been no case reports of HIT in double-positive patients. It is unclear whether this was incidental, but renal dysfunction due to HIT was less likely, since the improvement of renal function was accompanied by improvements of both titers and C-reactive protein. In the present case, the initial platelet count was very high, and the minimal platelet count was within normal limits $(26.9 \times$ $\left.10^{4} / \mu \mathrm{L}\right)$, presumably due to vasculitis-associated inflammation (19). Clotting in the dialyzer and extracorporeal circuit led us to suspect and diagnose the complication of HIT (15) before critical thrombosis occurred. HIT is a potential lifethreatening complication in all hemodialysis patients. Early clinical suspicion is essential, and careful attention is needed for those with systemic vasculitis, since they fundamentally have high platelet counts.

In conclusion, this report documents the successful recovery of renal function in a dialysis-dependent patient with MPA who developed double-positive RPGN and HIT. In younger patients who are better able to tolerate aggressive immunosuppression, even if their renal dysfunction is severe, optimal therapy appears to include plasma exchange and immunosuppression.

\section{The authors state that they have no Conflict of Interest (COI).}

\section{References}

1. Levy JB, Hammad T, Coulthart A, Dougan T, Pusey CD. Clinical features and outcome of patients with both ANCA and anti-GBM antibodies. Kidney Int 66: 1535-1540, 2004.

2. Rutgers A, Slot M, van Paassen P, van Breda Vriesman P, Heeringa $\mathrm{P}$, Tervaert JW. Coexistence of anti-glomerular basement membrane antibodies and myeloperoxidase-ANCAs in crescentic glomerulonephritis. Am J Kidney Dis 46: 253-262, 2005.

3. Lindic J, Vizjak A, Ferluga D, et al. Clinical outcome of patients with coexistent antineutrophil cytoplasmic antibodies and antibodies against glomerular basement membrane. Ther Apher Dial 13: 278-281, 2009.

4. Jayne DR, Gaskin G, Rasmussen N, et al. Randomized trial of plasma exchange or high-dosage methylprednisolone as adjunctive therapy for severe renal vasculitis. J Am Soc Nephrol 18: 21802188, 2007
5. de Groot K, Adu D, Savage CO. The value of pulse cyclophosphamide in ANCA-associated vasculitis: meta-analysis and critical review. Nephrol Dial Transplant 16: 2018-2027, 2001.

6. Warkentin TE. Heparin-induced thrombocytopenia: Pathogenesis and management. Br J Haematol 121: 535-555, 2003.

7. Jayne DR, Marshall PD, Jones SJ, Lockwood CM. Autoantibodies to GBM and neutrophil cytoplasm in rapidly progressive glomerulonephritis. Kidney Int 37: 965-970, 1990.

8. Hellmark T, Niles JL, Collins AB, McCluskey RT, Brunmark C. Comparison of anti-GBM antibodies in sera with or without ANCA. J Am Soc Nephrol 8: 376-385, 1997.

9. Serratrice J, Chiche L, Dussol B, et al. Sequential development of perinuclear ANCA-associated vasculitis and anti-glomerular basement membrane glomerulonephritis. Am J Kidney Dis 43: 26-30, 2004.

10. Yang R, Hellmark T, Zhao J, et al. Antigen and epitope specificity of anti-glomerular basement membrane antibodies in patients with Goodpasture disease with or without anti-neutrophil cytoplasmic antibodies. J Am Soc Nephrol 18: 1338-1343, 1997.

11. Levy JB, Turner AN, Rees AJ, Pusey CD. Long-term outcome of anti-glomerular basement membrane antibody disease treated with plasma exchange and immunosuppression. Ann Intern Med 134: 1033-1042, 2001.

12. Segelmark M, Hellmark T, Wieslander J. The prognostic significance in Goodpasture's disease of specificity, titre and affinity of anti-glomerular-basement-membrane antibodies. Nephron Clin Pract 94: 59-68, 2003.

13. Bosch X, Mirapeix E, Font J, et al. Prognostic implication of antineutrophil cytoplasmic autoantibodies with myeloperoxidase specificity in anti-glomerular basement membrane disease. Clin Nephrol 36: 107-113, 1991.

14. Warkentin TE, Chong BH, Greinacher A. Heparin-induced thrombocytopenia: toward consensus. Thromb Haemost 79: 1-7, 1998.

15. Yamamoto S, Koide M, Matsuo M, et al. Heparin-induced thrombocytopenia in hemodialysis patients. Am J Kidney Dis 28: 82-85, 1996.

16. Syed S, Reilly RF. Heparin-induced thrombocytopenia: a renal perspective. Nat Rev Nephrol 5: 501-511, 2009.

17. Carrier M, Rodger MA, Fergusson D, et al. Increased mortality in hemodialysis patients having specific antibodies to the platelet factor 4-heparin complex. Kidney Int 73: 213-219, 2008.

18. Roe SD, Cassidy MJ, Haynes AP, Byrne JL. Heparin-induced thrombocytopenia (HIT) and thrombosis in a haemodialysisdependent patient with systemic vasculitis. Nephrol Dial Transplant 13: 3226-3229, 1998.

19. Kirkland GS, Savige J, Wilson D, Heale W, Sinclair RA, Hope RN. Classical polyarteritis nodosa and microscopic polyarteritis with medium vessel involvement-a comparison of the clinical and laboratory features. Clin Nephrol 47: 176-180, 1997.

(C) 2011 The Japanese Society of Internal Medicine http://www.naika.or.jp/imindex.html 